Investigaciones Fenomenológicas, vol. Monográfico 5, 2015, 193-218.

e-ISSN: $1885-1088$

\title{
LA INDISCRETA PRESENCIA DE SÍ. LA GÉNESIS DE LAS HABITUALIDA- DES Y EL PROBLeMA DE LA REFLEXIÓn EN HUSSERL
}

\author{
The Indiscreet Presence of the Self. The Genesis of the \\ habitualities and the Problem of Reflection in Husserl
}

\author{
Roberto C.F. Menéndez \\ Universidad de Granada \\ Granada, España \\ roberto menendez@yahoo.es
}

\begin{abstract}
Resumen: Este trabajo tiene como objetivo presentar la cuestión de las habitualidades en la fenomenología de Husserl para pensar la génesis de la reflexión en nuestro mundo actual. En un primer momento, y principalmente a partir de libros primero y segundo de las Ideas relativas a una fenomenología pura y una filosofía fenomenológica, se revisarán el concepto de yo en Husserl en dos de sus dimensiones principales: el yo como polo idéntico de las vivencias y el yo como substrato de las habitualidades. Después realizaremos un análisis sobre la génesis de las habitualidades y su repercusión en el concepto de yo puro. Por último se ejemplificará esta cuestión en el caso de la reflexión, especialmente en el caso de una posible génesis negativa de la reflexión en forma de reflexividad ritual o hiperreflexividad. Con ello se quiere establecer una línea de conexión entre la subjetividad trascendental y nuestro mundo concreto, así como entre la fenomenología y la psicopatología.
\end{abstract}

Palabras clave: Yo puro, yo trascendental, habitualidades, hiperreflexividad.

\begin{abstract}
The aim of this work is to present the question regarding to the habitualities in Husserl's Phenomenology in order to think the genesis of reflection in our present world. In a first stage, and specially acording to the first and second book of Ideas [...] the concept of "I" will be considered in its two main dimensions: the I as identical pole of the livedexperiences and the "I" as substratum of habitualities. Afterwards, I will perfom an analysis on the genesis of the habitualities and its consecuences in the in the concept of pure I. In the last part this question will be ejemplied though the case of reflection in the form of a Ritual Reflectivity or Hypereflectivity. Whitin I would like to suggest the link conection between the trascendental subjectivity and our concrete world, and the phenomenology and psychopathology relationship as well.
\end{abstract}

Key Words: Pure I, Trascendental I, Habitualities, Hyperreflectivity. 


\section{$\S 1$. LA CUESTIÓN DEL YO COMO POLO DE LAS VIVENCIAS}

Quisiera aprovechar el centenario de la publicación del primer libro de las Ideas relativas a una fenomenología pura y a una filosofía fenomenológica para conmemorar especialmente aquellos momentos de la obra que quedaron abiertos y que forman parte de la reflexión sin fin que es todavía hoy la fenomenología. El hecho de que Husserl se decidiese a publicar esta obra, al contrario de lo que sucedió con los libros segundo y tercero, no impidió que muchos capítulos y temas quedaran solamente anunciados, a la espera de mejores determinaciones. Uno de los casos paradigmáticos es el del yo en sentido fenomenológico, pues al tiempo que Husserl marcaba aquí una diferencia fundamental con respecto a las Investigaciones lógicas, acusaba todavía una cierta inseguridad con respecto a su nueva postura, que se presentaba como una corrección obligada de la anterior. Celebrar la publicación de Ideas I es celebrar al tiempo la segunda edición de las Investigaciones lógicas, y no menos, la celebración de los libros segundo y tercero de las Ideas, que, aún no publicados, tuvieron su punto de partida en el fértil periodo que va desde 1909 hasta 1913, fecha de la publicación del primer libro.

Aunque Ideas relativas... se presenta como un manual de fenomenología, y como tal, marca un antes y un después en muchos desarrollos, no debe dejar de tenerse en cuenta que este punto y aparte, que cierra un ciclo y comienza otro, es también uno de los muchos puentes que dan testimonio del trabajo de fondo que supuso la vida filosófica de Edmund Husserl. Encontramos en su obra una constante revisión de los grandes temas de la fenomenología. Quizá el caso más emblemático es el de la epojé, sobre la que Husserl volvió casi obsesivamente. Otro caso será el que respecta al yo puro, el cual obligó a Husserl a correcciones importantes, como la que encontramos en segunda edición de las Investigaciones lógicas (1913). En la primera edición (1900-1901) la conciencia era entendida como una instancia anónima que por sus propios dinamismos daba unidad a las vivencias conformando una corriente unitaria. En la segunda edición Husserl reconocería el valor del yo como polo subjetivo de las vivencias. 
Lo hacía en una nota ${ }^{1}$, remitiendo a continuación al lector a las recién publicadas Ideas relativas... para una mayor profundización de la cuestión. ${ }^{2}$

En esta obra el problema se retoma en el parágrafo §57. En los apartados anteriores Husserl hace referencia principalmente a nociones como conciencia pura o conciencia trascendental. En las ocasiones en las que utiliza el término «yo», esta noción no es objeto de un tratamiento específico ${ }^{3}$. Por otro lado, a medida que se presenta el método de la reducción fenomenológica, se ve la necesidad de desconectar la validez del yo empírico y personal, poniendo en suspenso con ello nuestro concepto habitual del mismo. Esta desconexión o puesta entre paréntesis implica suspender la validez del cuerpo, lo que nos enfrenta a una cierta violencia que abre la pregunta sobre cómo seguir hablando de un yo. Husserl estaba especialmente interesado en los rendimientos esenciales de la conciencia, aquellos que no pueden ser desconectados. El concepto de yo puro no estaba todavía introducido. Es por ello que al llegar el momento de su tratamiento en el parágrafo citado, Husserl advertirá: «se presentan dificultades en un punto límite» ${ }^{4}$. Y añadirá, a continuación:

El hombre como ser de la naturaleza y como persona en el conglomerado personal, en el de la "sociedad", es desconectado; igualmente todo ser animal. Pero, ¿qué pasa con el YO PURO? ¿También el yo fenomenológico con que nos encontramos se ha convertido mediante la reducción fenomenológica en una nada trascendental? ${ }^{5}$

Este interrogante se presenta ya como detonante de una revisión de las tesis de las Investigaciones lógicas. Como hemos apuntado, en las Investigaciones el yo fenomenológico no era sino la síntesis que enlazaba todas las vivencias en la unidad de la corriente. Desde este punto de vista, la conciencia era entendida casi como una instancia anónima. En el giro que se da en Ideas I, y que se venía anunciando, al menos como problema, desde 1909-19106 , el lla-

\footnotetext{
${ }^{1}$ Husserl afirmaba en la primera edición, a propósito del yo puro en sentido fenomenológico: «he de confesar que no logro encontrar de ninguna manera ese yo primitivo, centro necesario de referencia». En la segunda edición añadiría una nota a este pasaje: «después he aprendido a encontrarlo; he aprendido a no dejarme extraviar, en la pura aprehensión de lo dado, por el temor a las degeneraciones de la metafísica» (HUSSERL, Edmund. Investigaciones lógicas, Madrid, Revista de Occidente, 1967; vol II, p. 165)

${ }^{2}$ Véanse especialmente los parágrafos $\S 4, \S 8$ y $\S 12$ de la 5 a investigación lógica.

${ }^{3}$ La idea de yo puro aparece en el parágrafo $\S 37$ y también en el $\S 46$, pero no es hasta el parágrafo referido que este yo puro se vuelve tema de una reflexión específica.

${ }^{4}$ HUSSERL, E. Ideas relativas a una fenomenología pura y a una filosofía fenomenológica. Libro primero: Introducción general a la fenomenología pura. México, Fondo de Cultura Económica, 2013; §57, p. 208 (En adelante, Ideas $I$ )

${ }^{5}$ Ibid

${ }^{6}$ Cfr. MARBACH, Eduard. Das Problem des Ich in der Phänomenologie Husserl, Den Haag, Martinus Nijhoff, 1974; pp. 59-87
} 
mado yo puro se hace cuestión, pero no al modo de algo que aparece a la conciencia, según la fórmula clásica de la intencionalidad. El yo no aparece como parte de las vivencias, y por lo tanto, no responde directamente al conocido principio de los principios que Husserl anuncia en el parágrafo §24 del texto que estamos comentando. El yo no es ni una vivencia ni parte ingrediente de una vivencia ${ }^{7}$. Su modo de descubrimiento y posterior explicitación comienza por exigir una atención propia, en el límite de la intuitividad fenomenológica.

Considerada por principio, cada cogitatio PUEDE cambiar, ir y venir, aunque puede dudarse si toda cogitatio es algo NECESARIAMENTE fugaz y no meramente algo FÁCTICAMENTE fugaz, como encontramos que es. Pero frente a esto parece ser el yo algo NECESARIO por principio, y en cuanto es algo absolutamente idéntico en medio de todo cambio real y posible de las vivencias, no puede pasar EN NINGÚN SENTIDO POR UN FRAGMENTO O MOMENTO INGREDIENTE de las vivencias mismas ${ }^{8}$

Husserl está determinado a no dejar que se confunda el yo con un objeto que pudiese aparecer en una vivencia, pero, por otro lado, ha de esclarecer el modo de su captación para que su posición no sea la de un principio heredado de idealismos de corte metafísico ${ }^{9}$. En un primer momento, su formulación no sólo no se distingue de la de Natorp, sino que llega a coincidir con la de Kant (El 'yo pienso' tiene que poder acompañar todas mis representaciones). Entre un principio inmanente absoluto - una "idea fija" - y un dato ingrediente de las vivencias, Husserl encuentra una solución que no deja de resultar extraña.

Si nos queda como residuo de la desconexión fenomenológica del mundo y de la subjetividad empírica inherente a éste, un yo puro ( $y$ entonces un yo por principio distinto para cada corriente de vivencias), entonces se nos ofrece con él una trascendencia SUI GENERIS - no constituida-, una TRASCENDENCIA EN LA INMANENCIA. Dado el papel inmediatamente esencial que desempeña esta trascendencia en toda cogitatio, no podremos someterla a una desconexión, aunque para muchas investigaciones las cuestiones del yo puro pueden quedan in suspenso ${ }^{10}$

Husserl lleva a cabo aquí una precisión muy notable de su método. En principio han de caer bajo la desconexión fenomenológica todas las trascendencias, incluida, por ejemplo, la de de Dios. El yo, entendido como una trascendencia, debería de caer también. Esta es la postura que se mantenía en las Investiga-

\footnotetext{
7 Cfr. HUSSERL, Edmund. Phänomenologische Psychologie. Vorlesungen Sommersemester 1925, Den Haag, Martinus Nijhoff, 1966; pp. 207-208 (En adelante, Hua 9)

${ }^{8}$ HUSSERL, E. Ideas I. Op. cit., §57, p. 208

${ }^{9}$ Cfr. TAGUCHI, Shigeru. Das Problem des ,Ur-Ich' bei Edmund Husserl, Dordrecht, Springer, 2006; pp. 61-69

${ }^{10}$ HUSSERL, E. Ideas I. Op. cit., §57, p. 209
} 
ciones lógicas, según la cual la unidad de las vivencias no era producida por algo a lo que le fuese conveniente la denominación de yo. Aquí, como vemos, y según la idea de una trascendencia sui generis que por su papel esencial no puede ser desconectada, parecemos asistir a un punto intermedio, a una suerte de puente en la explicitación del método fenomenológico. Husserl vincula la trascendencia del yo puro a la cogitatio, a toda cogitatio, o mejor, a la forma en que ésta hace aparecer sus objetos. Esto nos ayuda a avanzar la hipótesis según la cual, si bien el yo puro no es algo dado en la vivencia, sí es una remisión necesaria de la misma. En tanto trascendencia, el yo puede ser puesto entre paréntesis, desconectado. En tanto trascendencia sui generis, que no responde a lo dado en una vivencia, sino a lo remitido por ella como necesidad de principio, el yo no puede ser desconectado de modo absoluto, sino solo en el caso de algunas investigaciones. Pese a que en el campo trascendental se reconoce algo así como un yo puro, su presencia no es siempre relevante, y en muchos análisis fenomenológicos, especialmente aquellos que después se denominarán noemáticos, puede ser puesto en suspenso. Como decimos, Husserl mantiene todavía aquí una postura intermedia, al menos no definitiva. Es por ello que, si en la segunda edición de las Investigaciones nos remitía al primer libro de las Ideas, llegados ahora al mismo, y en este problema en concreto, Husserl nos remite al libro segundo, haciendo referencia, una vez más, a las «difíciles cuestiones del yo puro» ${ }^{11}$

El segundo libro, no publicado por determinación de Husserl, se pregunta precisamente por el modo de evidencia del yo fenomenológicamente reducido. Tal y como se expone el problema, el yo puro parece ser un momento de lo que Husserl denomina la conciencia pura. En otras palabras, no hay una coincidencia inmediata, sino más bien una co-dación (Mitegegebenheit). El yo no es dado como aquello que se da en una vivencia, es decir, como un objeto que lleva en sí un índice de trascendencia mundana. Husserl no hablará por tanto de lo dado (gegeben) para referirse al yo puro, sino de lo captado (erfasst). Lo que se anuncia entonces es una remisión que va de lo vivido a su concentración subjetiva. El yo no es un principio metafísico, «no es en modo alguno nada misterioso o aún místico. Yo me tomo como el yo puro en tanto que me tomo puramente como el que en el percibir está dirigido a lo percibido, en el conocer a lo co- 
nocido, en el fantasear a lo fantaseado, en el valorar a lo valorado, en el querer a lo querido; en toda ejecución de un acto yace un rayo del estar-dirigido que no puedo describir de otra manera más que diciendo que tiene su punto de partida en el "yo", el cual evidentemente permanece ahí indiviso y numéricamente idéntico»"12

El yo, entonces, no es solo lo que permanece idéntico independientemente de los muchos objetos que sean motivo de su atención; también ocurre en relación a los muchos actos que desempeña, lo que nos lleva a considerar una primera diferenciación metódica. Uno y el mismo yo, pero múltiples modos de afección y atención de lo dado ${ }^{13}$. Esto es importante porque el yo, en algún sentido, se diferencia de sus actos, y en tanto yo puro no supone todavía la coincidencia con el conjunto de los mismos. Más bien, el yo es una remisión que habita en todos ellos en tanto que todos forman parte de una y la misma corriente de conciencia ${ }^{14}$. En tanto que las cosas no aparecen simplemente ante la vista, sino que afectan de alguna manera, hablamos de un principio subjetivo «que despierta ante...», «que es afectado por...», «que lleva su atención a...». Esto se ve claramente en actos tales como el deseo, el amor, el odio, donde el sujeto, por así decir, no supone un mero reflejo del objeto, sino que marca una distancia con el mismo a través de tales actos. Ahora bien, el yo puro no es aquel que ahora ama u odia, como sujeto en el mundo. El yo puro apunta al polo unitario que subyace a pasiones tan aparentemente contrarias como el amor y el odio. Por un lado, el yo puro mienta el polo subjetivo de toda vivencia, es decir, su identidad y unidad numérica. Por otro lado, como veremos, se entiende ese polo como un centro deseante, es decir, como un centro activo y, más que pasivo, pasional, en tanto la afección no es neutral, sino que implica un principio de respuesta, una reacción.

En los actos del cogito polimorfo aislado o vinculado por él, el yo puro practica sus "funciones" puras, y en tal medida querríamos designar, en sentido traslaticio, los actos mismos como funciones. Aquí, el yo puro puede, por un lado, diferenciarse de los actos mismos en cuanto que funciona en ellos y se refiere a objetos a través de ellos; por otro lado, sin embargo, sólo puede diferenciarse de ellos abstractamente. Abstrac-

\footnotetext{
12 HUSSERL, Edmund. Ideas relativas a una fenomenología pura y una filosofía fenomenológica. Libro Segundo: Investigaciones fenomenológicas sobre la constitución, México, Fondo de Cultura Económica, 2005; §22, pp. 133-134 (En adelante, Ideas II)

${ }^{13}$ HUSSERL, E. Ideas II. Op. cit., §25

${ }^{14}$ Husserl propone esta relación intrínseca en los términos de «corriente de vivencias y de la necesaria referencia de la misma al yo puro». HUSSERL, E. Ideas I. Op. cit., §78, p. 257
} 
tamente, en tanto que no puede ser pensado como algo separado de estas vivencias, como algo separado de su "vida"15

En un primer momento, solo podemos tener acceso al yo puro a partir de una vivencia en la que algo aparece. En ella, dirigimos la mirada hacia aquel polo subjetivo que en el percibir decimos que percibe, sin confundirse con el yo empírico situado en el mismo espacio que la cosa percibida. La atención está puesta en la remisión subjetiva que, por así decir, no cae dentro del cuerpo. Este yo polo (Ichpol), como tal, es un momento de la estructura intencional, una remisión que constantemente puede ser descubierta por la reflexión; incluso en aquellas pasiones que parecen más anónimas. Pero esto solo se lleva a cabo sobre el fondo de una vivencia reducida. Sólo aquí el yo puro puede ser captado, es decir, convertido en término de una reflexión. Con todo, la objetivación resultante del yo no llega a vencer la última resistencia que éste supone, en virtud de la cual nunca puede ser puesto enteramente del lado del objeto: el polo subjetivo de la vivencia es mantenido también en reflexiones en las cuales se pone ante la vista ese mismo yo ${ }^{16}$. Por otro lado, el yo puro está cifrado en su identidad, de modo que se mantiene como el mismo independientemente de que ahora odie lo que antes amó o viceversa. La atención está puesta precisamente en que soy yo quien antes amó y ahora odia, en tanto que, aunque este cambio modifique mi yo personal, no modifica el carácter idéntico de yo puro.

\footnotetext{
Yo soy y era el mismo que, al durar, "impera" en éste y en cada uno de los actos de conciencia; aunque, por otro lado, no soy un momento efectivo del acto mismo a la manera de un fragmento integrante. No soy un momento efectivo: en esto hay que poner la atención particulamente. Todo cogito con todos sus fragmentos integrantes se origina o cesa en el flujo de las vivencias. Pero el sujeto puro no se origina ni cesa, aunque a su modo "entra en escena" y de nuevo "sale de escena". Entra en acción y queda de nuevo fuera de acción. Qué es esto y en general qué es y qué obra él mismo, lo captamos, o él lo capta, en el percibirse a sí mismo, que es justo una de sus acciones, y una acción tal que fundamenta la absoluta indubitabilidad de la captación del $\operatorname{ser}^{17}$
}

Tendríamos que decir, en pureza fenomenológica, que este yo no nace ni muere. Si hay experiencia de algo, y por tanto conciencia, hay un polo subjetivo que concentra esa experiencia, como un centro de irradiaciones que aúna la

\footnotetext{
15 HUSSERL, E. Ideas II. Op. cit., §22, p. 135

16 «por todas partes pueden en verdad diferenciarse lo objetivado y lo "primigeniamente" no objetivado, por ejemplo, el yo puro percibido y el percipiente». HUSSERL, E. Ideas II. Op. cit, §23, p. 138.

17 HUSSERL, E. Ideas II. Op. cit, p. 139
} 
afección y la atención. Ahora bien, ese yo idéntico, siempre presente en la corriente de vivencias, aunque no siempre presente a sí mismo, sí tiene diferencias en su modo de aparecer, y en su modo de participar. Si bien es absolutamente necesario, y como tal no puede pensarse una vivencia absolutamente desprovista de este carácter subjetivo, sí podemos hablar, gracias a la reflexión, de vivencias en las que el yo puro no participa, llegando a hablar incluso de vivencias sin yo (Ichlos). Son estas vivencias las que ponen a prueba el concepto más extremo de yo puro, en tanto que, sin participación, no se elimina ese carácter concéntrico que da unidad a las vivencias ${ }^{18}$. Podemos hablar, por tanto, de vivencias del un yo ausente, o mejor dicho, latente. Lo que se mantiene aquí son aquellos rendimientos de la conciencia por los cuales ésta salva su unidad pese a la no participación del yo. En todo caso, una reflexión ulterior podría descubrir siempre esta remisión de lo vivido de modo pasivo -lo que Husserl llama a veces la conciencia sorda - a un centro polarizador.

Es importante esta distinción, que Husserl no llega a establecer con claridad suficiente: por un lado, el yo como centro polar independiente de su participación y presencia ante sí. Función propia de síntesis pasivas, involuntaria, y en algún sentido, anónima. Por otro lado, yo como aquel que desea, que lleva su atención hacia... que se siente afectado o atraído por..., etc ${ }^{19}$. En el primer caso, como Husserl dirá en varias ocasiones, el yo puro es absolutamente simple, no entraña misterio alguno ni expresa una entidad metafísica. En nuestros términos consideramos que este concepto de yo resulta de la remisión genitiva de toda vivencia a un polo subjetivo.

Para profundizar un poco más esta cuestión volvemos ahora al primer libro de las Ideas relativas. Lo retomamos en el §80. Allí Husserl sigue manteniendo su interés en la conciencia trascendental, entendiendo que el yo puro es una suerte de remisión constante, y en la mayor parte de los casos latente, que acompaña a todo tipo de vivencias. La atención, no obstante, está puesta en las formas esenciales de vivencia y en los modos de llevarse a cabo en relación a sus objetos, es decir, en la relación entre el aparecer, lo que aparece y el

\footnotetext{
18 «Al yo puro le pertenece por consiguiente, en vez del originarse y el cesar, solamente la peculiaridad esencial de que tiene su presentarse y su ausentarse, de que comienza a funcionar actualmente, comienza a imperar, y termina de hacerlo» HUSSERL, E. Ideas II. Op. cit., p. 140

${ }^{19}$ HUSSERL, E. Hua 9, Op. cit., pp. 208-209
} 
sentido con que se aprehende. Con todo, se rescata aquí brevemente la atención a la cuestión del yo puro.

Husserl vuelve a preguntarse en qué sentido podemos seguir hablando de un yo después de llevar a cabo la desconexión fenomenológica ${ }^{20}$. Como hemos señalado, el yo se descubre siempre a partir de sus vivencias, no como un ingrediente de las mismas, sino como aquel que "vive" en ellas. No es posible descubrir al sujeto fuera de esta envoltura; como tal el yo viene a ser parte del horizonte interno de la corriente de las vivencias, un rendimiento constante y continuo del que nos da cuenta la remisión de las vivencias a un «viviente».

Pese a estos peculiares entrelazamientos con todas "sus" vivencias, no es el yo vivenciante nada que pueda tomarse POR SÍ y del que puede hacerse un objeto PROPIO de investigación. Prescindiendo de sus "modos de referencia" o "modos de comportamiento", está totalmente vacío de componentes esenciales, no tiene ningún contenido explicable, es en sí y por sí indescriptible: yo puro y nada más ${ }^{21}$

\section{§2. LA GÉNESIS DE LAS HABITUALIDADES}

Nos conviene detenernos aquí, porque Husserl vuelve a indicar involuntariamente en este pasaje la diferencia entre el yo puro como simple polo subjetivo, captable en todo momento por una reflexión como aquel centro subjetivo que permanece idéntico, y ese mismo yo puro en tanto que tiene modos de comportarse, sin que estos modos caigan todavía del lado del sujeto empírico. A la sencillez por la cual toda vivencia implica esta remisión subjetiva, se añadirá la complejidad de los modos en los que el yo puro se expresa. Como se ha advertido, el yo sería un centro de irradiación, de donde surgen rayos intencionales, pero también a donde llegan, en forma de afecciones que lo movilizan. A la identidad de su carácter polar se añadirá ahora la diferencia de sus muchos modos de atención, afección y comportamiento con respecto a lo dado en las vivencias y a las vivencias mismas. Esta diferencia en lo que al tratamiento me-

\footnotetext{
20 «El "estar dirigido a", "estar ocupado con", "tomar posición relativamente a", "experimentar, padecer", encierra NECESARIAMENTE en su esencia esto: ser, precisamente, un "desde el yo", o, en el rayo de dirección inverso, "hacia el yo" -y este yo es el PURO; a él no puede hacerle nada ninguna reducción». HUSSERL, E. Ideas I. Op. cit., §80, pp. 268-269

${ }^{21}$ HUSSERL, E. Ideas I. Op. cit., p. 269
} 
todológico se refiere no es destacada especialmente por Husserl, pero supone una diferencia esencial entre uno y otro carácter de uno y el mismo yo. ${ }^{22}$

Hemos anunciado aquel otro aspecto que refiere a las tomas de posición. Al entender el centro de irradiación como concreto, es decir, como diferenciado de otros sujetos, se enuncia un nuevo tema, que, al quedar restringido a la esfera trascendental, no considera todavía al yo como un sujeto empírico. Husserl lo enuncia del siguiente modo:

En el interior de una corriente de conciencia monádica absoluta se presentan ahora ciertas conformaciones de unidad, que son, empero, completamente diferentes de la unidad intencional del yo real y sus propiedades. De éstas forman parte unidades tales como las "MENCIONES" PERMANENTES de uno y el mismo sujeto. Se puede llamarlas, en cierto sentido, "habituales", pero no se trata de un hábito de costumbre, como si el sujeto empírico adquiriera disposiciones reales, que en tal caso se denominan disposiciones de costumbre. El hábito del que aquí se trata no pertenece al yo empírico, sino al puro. La identidad del yo puro no solamente radica en que yo (de nuevo el yo puro) puedo captarme, con respecto a todo cogito, como el yo idéntico del cogito; más bien: también soy, y a priori, el mismo yo, en tanto que en mis tomas de posición soy necesariamente consecuente en un sentido determinado ${ }^{23}$

Husserl hace aquí la diferencia entre estas dos referencias al yo en sentido fenomenológico: por un lado el yo idéntico, como polo subjetivo para todas las vivencias: centro de irradiación de atenciones y afecciones. Por el otro, un yo que en sus tomas de posición, en su quedar afectado de una determinada manera, en el atender de un cierto modo, mantiene un estilo que genera lo que se llamará habitualidad. Con este concepto Husserl diferencia estas modificaciones intencionales de aquellas otras que responden a nuestra actitud en el mundo, y que Ilamamos hábitos o habilidades. El concepto de habitualidad cae y se queda en el ámbito ganado tras la reducción trascendental, pero habrá que atender con más precisión a su génesis. En general Husserl entiende que los mismos motivos favorecen las mismas tomas de posición, que a su vez conllevan una cierta orientación de la atención, una cierta tonalidad emocional, un género de juicios, etc. Existe una regularidad que se va generando en vistas a similares motivaciones. En todo caso podemos captar una y la misma dirección intencio-

\footnotetext{
22 «El yo es el sujeto idéntico de la función en todos los actos de la misma corriente de conciencia; es el centro de irradiación, o centro de recepción de radiación, de toda vida de conciencia, de todas las afecciones y acciones, de todo atender, captar, referir, vincular, de todo tomar posición teórico, valorativo, práctico, de todo estar alegre y estar triste, esperar y temer, hacer y padecer, etc. Con otras palabras, todas las polimorfas (vielgestaltigen) particularidades de la referencia intencional a objetos que se denominan actos, tienen su necesario terminus a quo, el punto-yo, del cual irradian». HUSSERL, E. Ideas II. Op. cit., §25, pp. 141-142

${ }^{23}$ HUSSERL, E. Ideas II. Op. cit., §29, p. 148
} 
nal que engloba toda esta suerte de menciones. Al igual que en el caso del yo puro, que puede no estar presente, estas habitualidades pueden no presentarse, y sin embargo se mantienen como las mismas: por ejemplo, una fruición particular que sentimos cuando llega el otoño. En el resto de las estaciones este rendimiento se mantiene en estado latente, pero resurge a la llegada de un nuevo otoño. Hay un elemento que se reconoce como el mismo, pese a sus eventuales modificaciones.

Si esto es así, conviene precisar de nuevo la relación que guarda el yo como polo idéntico con el yo que, al verse «afectado por...», genera una cierta permanencia de esta su forma de «ser afectado». Si hablamos de yo puro para el yo entendido como polo idéntico, y de yo trascendental para el yo de las habitualidades, habrá que decir que la diferencia entre ambos, en un sentido preciso, es diametralmente opuesta. Mientras que el yo puro, entendido como polo subjetivo que comprende y concentra la actividad y pasividad del sujeto, es captado en una reflexión simple, y es captado como el mismo en cualquier momento de la corriente de conciencia, el yo trascendental, en cambio, es explicitado de un modo infinito, y en cada nueva determinación tenemos delante un yo más rico, lo que expresa una diferencia que fluctúa de lo desconocido a lo conocido. La explicitación del yo trascendental no tiene fin, como Husserl nos recordará en las Meditaciones cartesianas ${ }^{24}$. En este sentido, no podemos afirmar que cada vez que volvemos sobre él estamos captando una y la misma cosa, pues lo captamos siempre en nuevos actos y en relación a rendimientos que, si bien no son inéditos, se renuevan siempre de un modo original. Husserl hará referencia a esta complejidad que resalta con la simplicidad del yo puro entendiendo que el yo trascendental hace referencia a formas fundamentales de intencionalidad, tales la percepción, la memoria, la fantasía, el juicio, el deseo, etc. y a los modos en que estos rendimientos, al llevarse a cabo en actos

\footnotetext{
${ }^{24}$ Husserl no cifra su interés en fundamentar el conocimiento en el yo pienso cartesiano, que en nuestra terminología haría referencia al yo puro indubitable que acompaña a cada vivencia. En efecto, este yo puro ha de ser comprendido como polo subjetivo del yo trascendental, que es comprendido a partir de un campo infinito de experiencia: «En contraste con Descartes, nosotros nos sumimos en la tarea de explorar el campo infinito de la experiencia trascendental. La evidencia cartesiana, la de la proposición ego cogito, ego sum, no da su fruto porque Descartes no sólo omite el poner en claro el puro sentido metódico de la epojé trascendental, sino que también omite el dirigir la mirada a la posibilidad que tiene el ego de explorarse a sí mismo hasta lo infinito y sistemáticamente por medio de una experiencia trascendental, y con ello al hecho de que el ego está ahí presto, como un posible campo de trabajo» (HUSSERL, Edmund. Meditaciones cartesianas, México, Fondo de Cultura Económica, 2005; p. 73)
} 
concretos, generan unas habitualidades que identifican a cada yo frente a los otros.

Es preciso reconocer que Husserl no hace diferenciaciones claras entre los títulos de yo puro y el yo trascendental, que en muchas ocasiones utiliza indistintamente $e^{25}$. No obstante, si identificamos al yo puro con el polo subjetivo al que remite toda vivencia, absolutamente simple y que cae fuera del tiempo de la corriente de conciencia, se hace necesario otro calificativo para determinar al yo -más bien, a aquel aspecto del yo- que, al ser afectado, genera una permanencia de la forma de afección; hablamos para este caso de una historicidad, que sin embargo no cae todavía del lado del sujeto empírico. En nuestro caso reservamos para esta otra concepción del sujeto el calificativo de yo trascendental. Este sujeto sería aquel que se manifiesta en sus actos, los cuales generan o renuevan ciertas habitualidades, horizontes y remisiones intencionales de todo tipo, las cuales no pueden tenerse por idénticas en el mismo sentido en que tenemos por idéntico al yo puro. Así, mientras éste se capta en un acto simple, el otro ha de ser explicitado hasta el infinito, pues aún diferenciados y explicitados los actos esenciales y específicos que encontramos bajo los títulos sugeridos - percepción, memoria, imaginación, juicio, espera, etc. - queda todavía entrar en sus muchas sutilezas, que nunca se dejan determinar de modo absoluto $^{26}$. Este yo trascendental se vincula sobre todo a la experiencia, que es experiencia de mundo y sobre todo experiencia en el mundo.

Antes de avanzar por este lado, permítasenos hacer una nueva referencia a los puntos comunes entre las llamadas habitualidades y el carácter polar de la experiencia. Pues no se trata de oponer el yo puro al yo trascendental, sino de comprender su diferencia al interior del método fenomenológico. Uno y otro son el mismo, pero, mientras que el yo trascendental se determina en un campo que ha de ser pensado como histórico, el yo puro, en su simplicidad, cae fuera del tiempo, no se historiza. Esta tesis diferencia dos aspectos de la subjetividad trascendental en general al tiempo que reconoce su implicación mutua. En rea-

\footnotetext{
25 Pese a que Husserl utiliza indistintamente en muchas ocasiones las nociones de yo puro y yo trascendental, hay que notar que la primera de las opciones es sobre todo utilizada para referirse al carácter idéntico de la subjetividad, el yo como polo. En cambio, reserva el concepto de yo trascendental cuando hace referencia a aquel campo de experiencia infinito que la fenomenología tiene como cometido explicitar. Pese a ser en todos los casos uno y el mismo sujeto, esta distinción metódica se aprecia en los parágrafos $\S 31$ y $\S 32$ de las Meditaciones cartesianas, donde Husserl diferencia «el yo como polo idéntico de las vivencias» de «el yo como sustrato de habitualidades».

${ }^{26}$ Cfr, HUSSERL, Edmund, Grundprobleme der Phänomenologie 1910/11, Den Haag, Martinus Nijhoff, 1977, §28; p. 69.
} 
lidad ambas referencias a un yo operan en niveles diferentes. Husserl llega a decir que el yo como polo idéntico de todas las vivencias no debería de ser siquiera llamado con ese título si lo que queremos es pensar la remisión polar que no se temporaliza ${ }^{27}$. El yo como puro polo sería completamente anónimo, requerido de afección para poder mostrar aquel carácter suyo que va más allá de la polaridad idéntica, y que refiere a un sujeto capaz de temporalizarse. Dicho de otro modo, al polo idéntico de las vivencias no le convendría la denominación de yo si este polo no se viese afectado por impresiones que generan una reacción por su parte, volviéndolo un sujeto histórico ${ }^{28}$. Una vez más, pese a que se hablemos de uno y el mismo yo -el concepto englobante sería el de subjetividad trascendental- la diferencia entre el carácter polar no-temporal y el carácter histórico del yo afectivo y activo es fundamental en términos metodológicos.

Ambas referencias tienen en común el carácter genitivo al que ya hemos aludido. Las vivencias son mías, y las tomas de posición, los modos de afección o atención, por seguir con el ejemplo que hemos empleado, son también, en un sentido no empírico, míos ${ }^{29}$. Cuando hablamos de habitualidades no debemos confundirlas con las habilidades personales, fruto de la fuerza de voluntad y del ejercicio continuado. $Y$ sin embargo, pese a que Husserl quiere reservar el concepto de habitualidad para un tipo de adquisición permanente propia de la esfera trascendental $-y$ por lo tanto no empírica-, no se puede negar que las habitualidades, como habitualidades del yo puro, se han generado en el mundo de la vida que tras la reducción ha quedado en suspenso. Husserl pone su interés en el reconocimiento de la identidad y la permanencia las tomas de posición que el sujeto adquiere, y que también suponen un estilo a la hora de percibir, recordar, imaginar $y$, como veremos, reflexionar ${ }^{30}$. El tipo de tenencia de

\footnotetext{
27 HUSSERL, E. Die "Bernauer Manuskripte" über das Zeitbewußtsein (1917/18), Kluwer Academic Publishers, Dordrecht, Netherlands, 2001; pp. 277-288.

${ }^{28}$ Husserl dirá a propósito que el «yo centrípeto no es un vacío polo de identidad (no más que lo es ningún objeto), sino que, en virtud de una ley de la "génesis trascendental", gana una nueva propiedad duradera con cada acto de un nuevo sentido objetivo irradiado por él» (HUSSERL, E. Meditaciones cartesianas, op. cit., p. 111)

${ }_{29}$ «No se trata aquí del contenido doquiera idéntico de la convicción en cuanto unidad ideal, sino del contenido en cuanto algo idéntico PARA EL SUJETO, en cuanto algo suyo propio, obtenido por él en actos anteriores, pero no efímero como los actos, sino inherente al sujeto que dura como lo permanente que dura para él» HUSSERL, E. Ideas II. Op. cit., §29, p. 153

${ }^{30}$ Hay que notar que las habitualidades de las que trata Husserl, aún reservadas a la esfera trascendental, van a dar paso al yo personal a partir de análisis que el autor considera de orden genético. En este sentido leemos en las Meditaciones cartesianas: «En cuanto el yo, en virtud de una propia génesis activa, se constituye en sustrato idéntico de duraderas propiedades privativas de un yo, se constituye consiguientemente en un yo personal "estable y duradero", en un amplísimo sentido que permite hablar tam-
} 
estas posiciones permanentes no deben confundirse entonces con una posesión, como lo aquello que tengo. En tanto que son posiciones en las que vivo, se entienden como un "haber". Es importante comprender bien este carácter genitivo subjetivo de la corriente de las vivencias y del estilo unitario que en ella se mantiene.

La actividad, es decir, la participación del yo, la toma de posición, deviene pasividad en la habitualidad que mantiene esa posición cuando "su" objeto ha desaparecido. El primer momento puede entenderse como afectivo-reactivo, y de él emana una pasividad que resulta secundaria con respecto a la pasividad primera que es aquella de la predonación objetiva. La habitualidad resultante, nos dirá Husserl, pertenece a la subjetividad trascendental. No puede ser desconectada, y sin embargo, su génesis proviene del mundo de la vida y de las disposiciones naturales que nos vincularon al mismo. De este modo, el mundo, en su forma general y como entretejido por una estructura de afección, "cala" hasta la subjetividad trascendental a través de las habitualidades. Éstas exigen una sedimentación y también una reactivación, lo que nos recuerda la posibilidad, para el yo puro, de entrar y salir de escena. Veremos que esta reactivación, unida a la posibilidad de una permanencia del yo en la escena de sus vivencias, nos permitirá anunciar la posibilidad de habitualidades cuya reactivación no es voluntaria, sino más bien obsesiva, o en otros términos, ritual. Lo que planteamos, de momento, es la necesidad de vincular las habitualidades a las relaciones con los "objetos" que las generan, poniendo la atención en el mundo de la vida. Si éste puede ponerse entre paréntesis en su actualidad, no puede, sin embargo, en cuanto a la relación histórica que mantengo en él y que ha generado dichas habitualidades.

Estas consideraciones nos ponen ante una problemática a la que tarde 0 temprano cualquier investigador en fenomenología se ve enfrentado: se trata de la conjugación entre la subjetividad trascendental y el carácter históricofactual de cada sujeto. En especial, interesa aquí comprender bien los límites de la reducción, en la medida en que la suspensión de la validez del mundo no consiste sino en la revelación del carácter histórico del sujeto, de su ser afecti-

bién de "personas" infrahumanas. Y aun cuando las opiniones en general sólo son relativamente duraderas, y tienen sus modos propios de "mutación" (tomando distintas modalidades las posiciones activas, "borrando" o negando, aniquilando su validez), el yo acredita en medio de semejantes mudanzas un estilo permanente, un "carácter personal"» (HUSSERL, E. Meditaciones cartesianas, op. cit., p. 112) 
vo y su consiguiente individuación histórica en un mundo concreto. Aunque tratemos de descubrir el eidos ego, como Husserl dirá en numerosas ocasiones, ello no implica una desatención a su carácter histórico, sino más bien el reconocimiento de que una fenomenología eidética es una fenomenología concreta. La conciencia que trata de elaborar una eidética de las vivencias no puede poner al ego que las soportaría sin partir de su condición de mónada concreta, o lo que es aquí lo mismo, su condición de subjetividad habitual, cuyas potencialidades no resultan indeterminadas. Como Husserl anotará en un manuscrito, «el eidos del ego trascendental es impensable sin el yo trascendental fáctico». ${ }^{31}$

La experiencia que resulta de la atención reflexiva a las habitualidades es la del descubrimiento de una correlación no empírico-causal entre el sujeto y el mundo. No solo hay una correlación entre los mismos motivos y las mismas posiciones. También aquellos nuevos objetos y situaciones que recuerdan a otros generan posiciones que se asimilan a otras anteriores. La puesta en suspenso de la validez del mundo y de las posiciones dóxicas permite precisamente rastrear la génesis de estas habitualidades y sistemas asociativos que en la disposición natural pasan desapercibidos. La fenomenología suspende la correlación causal con el mundo para hacer emerger la correlación en términos de sentido. Ahora bien, ¿qué hacer en el caso de habitualidades que imposibilitan u obstaculizan la reflexión fenomenológica? ¿Cómo entenderlas a partir de la epojé?

Como conclusión de este apartado, entendemos que lo importante es la consideración de las habitualidades como pertenecientes al yo trascendental, y no meramente al yo empírico. Decimos entonces que las habitualidades "calan" desde el mundo de la vida hasta el sujeto trascendental, que de este modo se historiza. Para ello hay que entender que el polo subjetivo no es un mero cen-

\footnotetext{
31 HUSSERL, Edmund, Zur Phänomenologie der Intersubjetivität (1929-1935). Den Haag, Martinus Nijhoff, 1973, p. 385: «Das Eidos traszendentales Ich ist undenkbar ohne trascendentales Ich als faktisches». En realidad, no hace falta irse a un manuscrito de Husserl para encontrar estas ideas. También en las Meditaciones Cartesianas encontramos esta afirmación sobre la concreción del ego, incluyendo las habitualidades: «la evidencia absoluta del ego sum alcanza por necesidad a la variada experiencia de la vida trascendental y a las propiedades habituales del ego, aunque sólo sea dentro de ciertos límites [...] Indicado de una manera todavía más exacta, acaso haya que indicar lo siguiente. No es la vacía identidad del "yo soy" el contenido absolutamente indubitable de la experiencia trascendental del yo, sino que a través de todos los datos particulares de la experiencia real y posible del yo se extiende una estructura universal y apodíctica de la experiencia del mismo [...] Con esta estructura está en conexión, y a ella misma es inherente también, el que el yo esté bosquejado apodícticamente para sí mismo como un yo concreto y dotado de un contenido individual de vivencias, disposiciones; bosquejado dentro de un horizonte de cómo un objeto accesible a lo largo de una posible experiencia de sí mismo, susceptible de perfeccionarse in infinitum». HUSSERL, E. Meditaciones cartesianas. Op. cit., pp. 70-71
} 
tro de irradiación, sino un centro afectivo en donde la obligada respuesta a las afecciones permanece de algún modo. A ello añadimos que las habitualidades no provienen sino de la experiencia subjetiva del mundo. Las habitualidades se generan en la historia del sujeto, lo que introduce la referencia al mundo de la vida que, al igual que el sujeto, es un mundo concreto a diferencia de otros mundos posibles. Al carácter afectivo del sujeto se une la particular retención de estas afecciones: no es solo que se recuerde lo vivido. Se generan y mantienen con ello modos de afección, de atención, de respuesta, de interacción. En otros términos, la experiencia da lugar a la génesis de una sensibilidad y de un estilo. $^{32}$

\section{§3. LAS HABITUALIDADES RESPECTO DEL PROBLEMA DE LA REFLEXIÓN}

A partir de estas consideraciones se abre una cuestión delicada en la fenomenología de Husserl, que es la de la autoconstitución del sujeto en el mundo. En un primer momento, a Husserl le interesa la constitución del mundo por los rendimientos de la subjetividad trascendental. En este proceso el sujeto se constituye a sí mismo, se da a sí mismo una forma unitaria y perdurable. En esta constitución debida a diferentes rendimientos y síntesis pasivas entran en consideración las habitualidades, como hemos visto, y según las cuales el sujeto se constituye de modo personal. Husserl establece en todo momento la diferencia entre sujeto trascendental, aquel que entra en consideración tras la reducción fenomenológica, y el sujeto personal. Ambos son el mismo, y la fenomenología entiende principalmente al segundo como constituido por el primero. Pero en el caso de las habitualidades, éstas no pueden sino generarse en el mundo de la vida, constituyendo con ello un sujeto personal en un sentido que interesa al análisis fenomenológico. La cuestión de las habitualidades es uno de los puntos por donde Husserl deja pensar la posibilidad del sujeto trascendental como constituido, y no como constituyente.

\footnotetext{
32 En la corriente de conciencia «las vivencias anteriores no han desaparecido sin dejar huella; cada una de ellas sigue teniendo efectos. A la esencia del alma pertenece una continua innovación o transformación de disposiciones bajo los conocidos títulos de asociación, costumbre, memoria, también cambio motivado de parecer, cambio motivado de convicciones, de direcciones afectivas [...], de direcciones volitivas, las cuales, conforme al sentido de la aprehensión, no son sin duda reducibles a una mera asociación». HUSSERL, E. Ideas II. Op. cit., p. 175. Insisto de nuevo en la relación entre el mundo de la vida que conjuga todas estas posibilidades y el sujeto que, en su despliegue histórico, ve como las habitualidades que le constituyen calan hasta la esfera trascendental, no pudiendo ser desatendidas tras la puesta entre paréntesis de la realidad efectiva del mundo.
} 
Husserl hace referencia a la génesis de las habitualidades a través de leyes de motivación entre vivencias. Hay motivaciones de muchos tipos, relacionadas con la volición, con el deseo, con la razón, etc. Es importante recordar que estas motivaciones no siempre se destacan, así como tampoco lo hacen las asociaciones que pasivamente vinculan unas vivencias a otras. De este modo, la génesis de las habitualidades es eminentemente una génesis pasiva, y las remisiones que operan en un momento dado y que dependen de esas génesis pasan en muchos casos inadvertidas. Algo que experimento ahora me produce agrado debido una asociación con una experiencia similar. Ahora bien, en el momento en que pruebo este agrado, tales relaciones de asociación y motivación están disimuladas. Tenemos entonces habitualidades generadas por motivaciones de razón pero también por motivos emocionales, volitivos, etc. Hay que incluir, en último término, la ley más básica, que es la de la costumbre ${ }^{33}$.

El caso que ahora queremos ilustrar a propósito de las habitualidades es el de la reflexión, por ser ésta un rótulo para un tipo de actos que están estrechamente vinculados con la posibilidad de la fenomenología en general. Podríamos añadir también la fantasía, en tanto es también no solo un género de actividad de la conciencia en general, sino un tipo de actos propios del método fenomenológico, aquellos llamados «variaciones imaginativas». La cuestión, en ambos casos, versa sobre la posibilidad de que estas actividades - reflexión y fantasía - se configuren de un modo particular en la vida concreta del sujeto, generando habitualidades que, lejos de acercarnos a la posibilidad de la fenomenología, nos alejen de ella, por exceso o por defecto. Tomando como modelo la reflexión, diremos en primer lugar que su tratamiento supone otro de los anuncios de Husserl en el primer libro de las Ideas. Aunque él considera la reflexión como instrumento metódico, deja sin considerar todo el orbe de tipos de reflexiones que pertenecen a la vida de conciencia en general ${ }^{34}$. Este capítulo anunciado nos lleva a considerar no sólo las reflexiones naturales o psicológicas que llevamos a cabo en el mundo de la vida, sino ulteriores reflexiones de tipo

\footnotetext{
${ }^{33}$ cf. HUSSERL, E. Ideas II, §56: «La motivación como ley fundamental de la vida espiritual».

34 «La reflexión es [...] un título para actos en los cuales la corriente de vivencias con todos sus múltiples sucesos (momentos de las vivencias, elementos intencionales) llega a ser apresable y analizable con evidencia. La reflexión es, así podemos también expresarlo, el título del método de conciencia para el conocimiento de la conciencia en general. Pero precisamente en este método se vuelve ella misma objeto de posibles estudios: la reflexión es también el título para especies de vivencias esencialmente conexas, o sea, el tema de un capítulo principal de la fenomenología. En él, la tarea consiste en distinguir las diversas "reflexiones" y analizarlas íntegramente en orden sistemático». HUSSERL, E. Ideas I. Op. cit., §78, p. 254
} 
fenomenológico. La cuestión es si podría incluirse un capítulo de reflexiones patológicas, o como Husserl diría, anormlales: reflexiones habituales que por exceso de repetición acabarían volviéndose rituales.

Hemos dicho que las habitualidades se generan en el mundo de la vida. Éstas están en estrecha relación con las capacidades, que pertenecen a la índole normal del sujeto. Husserl realiza esta relación entre capacidad y normalidad en el parágrafo $§ 59$ de Ideas II, entendiendo que las capacidades, como potencialidades positivas, son el fruto de ulteriores habitualidades. Husserl apelará en innumerables ocasiones a la expresión «yo puedo» para hacer referencia a estas capacidades. Una de ellas, como queremos dar a entender, es la capacidad de la reflexión, generadora de habitualidades ligadas a la misma. Así lo leemos en un pasaje de La Crisis de las ciencias europeas, en el que Husserl afirma que «toda actividad y por consiguiente también la reflexiva produce sus adquisiciones habituales» 35 .

El problema que queremos plantear en este trabajo tiene que ver con los modos en que en el mundo se llevan a cabo las capacidades ligadas a la reflexividad humana, especialmente en lo que respecta a sus posibles modos de sedimentación. Si toda habitualidad surge a partir de la sedimentación y de la reactivación de una capacidad trascendental, ¿es posible que una reactivación excesiva de una capacidad llegue a extenuarla por completo, convirtiendo la habitualidad en ritualidad? En la medida en que la capacidad se actualiza con respecto a motivaciones, pudiera ser que el mundo en que el sujeto vive motivase reflexiones de un tipo concreto que acabasen por saturar esa misma potencialidad, orientándola hacia un único género de actualizaciones. Las habitualidades, como hemos visto, forman parte de disposiciones latentes del sujeto, de tomas de posición permanentes respecto a objetos, de asociaciones entre vivencias, etc. En la mayoría de los casos estas habitualidades forman parte del haber oculto del sujeto, y solo mediante la epojé son sacadas a la luz. Tanto si ejercen sus funciones en una percepción actual como si permanecen en estado latente, tales disposiciones sedimentadas ejercen sus funciones sin destacarse, es decir, sin llamar la atención del sujeto sobre las mismas. Tales habitualidades se reactivan con la experiencia, pero de un modo inadvertido. ¿Es posible

\footnotetext{
35 HUSSERL, Edmund. Las crisis de las ciencias europeas y la fenomenología trascendental. Buenos
} Aires, Prometeo libros, 2008, p. 151 
sin embargo una reactivación continuada de una disposición que llegue a perder este carácter disimulado, es decir, que llegue a desorientar la atención normal del acto intencional hacia su habitual objeto? En el caso de la reflexión habría que preguntar: ¿Es posible una reflexión, por ejemplo la del que sujeto que se vuelve sobre sí a partir de un sentimiento de culpabilidad, que llegue saturar la capacidad de reactivación de la potencia reflexiva, no permitiéndole volver sobre sí más que en los términos de un juicio moral? La respuesta no puede dejar de reconocer que las habitualidades se generan en un mundo concreto. Un mundo, en este caso, donde las circunstancias del sujeto le llevan a sentirse culpable.

Un difícil problema se vislumbra: el de la normatividad de la subjetividad trascendental tal y como se describe después de la reducción. Más arriba hemos hecho alusión a un factum de la conciencia: el yo puro, que es idéntico en tanto polo de las vivencias, «entra y sale de escena». Este dato viene a indicar una cierta normatividad. Si el yo puro puede entrar y salir de escena, es porque a su esencia no le corresponde ni estar siempre presente, ni estar siempre ausente. Podemos imaginar sin embargo un tipo de reflexividad en la cual el sujeto está siempre presente a sí mismo como polo subjetivo de las vivencias. En tanto es posible aprehender al yo puro como tal en una reflexión, lo que pertenece a su propia capacidad (yo puedo reflexionar y hacer de mí el tema de una percepción), es pensable una aprehensión continua en la que el carácter subjetivo de la vida estuviera siempre presente, impidiendo la realización normal de la intencionalidad, que tiene como término objetos del mundo ${ }^{36}$. Habríamos de una habitualidad pasiva, no voluntaria, forzada por la costumbre. Husserl hablaría aquí del paso de la normalidad a la anormalidad.

El problema así enunciado podría parecer un problema de psicología que en nada concierne a la fenomenología. Ésta, en efecto, no se ocupa de accidentes particulares, sino de estructuras esenciales de la experiencia como es la alucinación con respecto a la percepción. Pero aquí preguntamos precisamente por una reflexión que no es en ningún sentido accidental. La reflexión es una po-

\footnotetext{
${ }^{36}$ Husserl habla del modo normal y natural de la intencionalidad en estos términos: «El primer modo, el naturalmente normal que debe preceder incondicionalmente no por razones casuales sino esenciales, es el modo directo-hacia los objetos dados en cada caso, por lo tanto, en el vivir integrado en el horizonte de mundo, y eso en una constancia normal ininterrumpida, en una unidad sistemática que atraviesa todos los actos. Esta vida con orientación directa normal hacia los objetos dados en cada caso quiere decir: todos nuestros intereses tienen su fin en objetos» (HUSSERL, Edmund. Las crisis de las ciencias europeas, op. cit., p. 185)
} 
tencialidad de la subjetividad trascendental. Su función, en tanto tiende a observar al propio sujeto desde una suerte de distancia, tiende también a ganar conocimiento en lo que a sus rendimientos intencionales se refiere, aquellos que hacen que el mundo y las cosas aparezcan en un horizonte de sentido. En este sentido, la reflexión no aparece como un accidente, sino como una potencia de la vida de conciencia. Ahora bien, habría que hablar de reflexividad para hablar de la capacidad que da lugar a los diferentes tipos de reflexiones que Husserl menciona: reflexión natural, psicológica, fenomenológica, etc.

El problema, a nuestro juicio, exige pensar un mundo -el nuestro- en el cual la capacidad de actualizar la potencialidad reflexiva deje de ser una actividad libre del yo. Ya hemos dicho que las habitualidades no pueden sino generarse en el mundo de la vida. Esta constatación fuerza al fenomenólogo a tener que pensar el mundo concreto en el que vive y las motivaciones que éste ejerce sobre aquellas tendencias que el sujeto alberga como suyas. Husserl contempla en ocasiones la posibilidad de que al sujeto dejen de asistirle motivaciones racionales y sea privado de su libertad por ciertas afecciones ${ }^{37}$. Una vez más, aquí quiere avanzarse el pensamiento de una deriva histórica de la reflexividad humana que acabaría por ritualizar un tipo de reflexión psicológica, obsesiva, que haría perder el sentido de la reflexión filosófica. Esta preocupación podría plantearse como una cuestión metafenomenológica, en la medida en que no pone el mundo en suspenso sino que lo inspecciona desde el punto de vista de su influjo en la génesis del sujeto personal. El fenomenólogo tiene que desconectar estas posibilidades del mundo fáctico, pero le preocupan igualmente en la medida en que ejercen una resistencia a la posibilidad misma de la fenomenología. En su tiempo Husserl realizó un análisis de las ciencias positivas que exigió una suerte de estudio histórico de las mismas. Husserl llega a reconocer que la fenomenología exige para su desarrollo como disciplina un momento histórico preciso. En este caso preocupa pensar que un tipo de reflexión ritual pueda saturar la potencialidad reflexiva de la conciencia, impidiendo la actuali-

\footnotetext{
37 «En la experiencia se distingue, según su carácter fenomenológico, el "yo puedo" y el "yo no puedo". Hay un hacer sin resistencia, o una conciencia del poder sin resistencia, y un hacer en la superación de una resistencia, un hacer con un "contra" y una conciencia inherente del poder que supera la resistencia. Hay (siempre fenomenológicamente) una gradualidad de la resistencia y de la fuerza de superación: de la fuerza "activa" frente a la inerte de la resistencia. La resistencia puede volverse insuperable: entonces topamos con el "no hay manera", "no puedo", "no tengo fuerzas"» (HUSSERL, E. Ideas II, op. cit., p. 306)
} 
zación de aquella actividad que es la propia de la fenomenología, y también de la filosofía.

Es preciso reconocer que haría falta una reflexión ulterior para reconocer y estudiar esta reflexividad ritual o hiperreflexividad, pero el problema estriba en saber si esta posibilidad de derecho es siempre una posibilidad de hecho. Husserl respondería que es siempre posible en principio una reflexión sobre la reflexión, pero aquí nos preguntamos por un mundo que viniese a extenuar la reflexividad, no de un modo accidental, afectando a algunos sujetos, sino de un modo estructural, incidiendo en la comunidad intersubjetiva trascendental. Es claro que en principio una reflexión es siempre posible, lo que incluye una reflexión sobre otra reflexión. Lo que está en juego es que un sujeto aquejado de esta reflexividad ritual, que satura el orden de la potencialidad, sea capaz de llevar a cabo una reflexión ulterior, propiamente fenomenológica. La fenomenología suspende la actitud natural en la medida en que este es su punto de partida. La actitud natural supone un momento histórico que puede ser más o menos propicio al ejercicio de su suspensión. Husserl reconoce la delicadeza y complejidad de una correcta comprensión de la fenomenología, reconociendo que ciertos factores históricos ligados al desarrollo y la popularidad de las ciencias y la cultura pueden obstruir la labor de seguir correctamente los pasos de su método. Poniendo en relación la posibilidad efectiva de una mala comprensión o interpretación del método y finalidad de la fenomenología, Husserl dice en la Crisis que «el éxito efectivo de una filosofía trascendental depende hasta el extremo de la claridad de la toma de conciencia de sí mismo» ${ }^{38}$. No cabe duda de que aquí la reflexión psicológica de los sujetos está en estrecha relación con la posibilidad de la reflexión fenomenológica. Por ello, puede decirse que si bien la fenomenología es siempre posible por razones de esencia, la misma fenomenología, en referencia a la circunstancia histórica del sujeto que ha de llevarla a cabo, es también una ciencia frágil.

Si nos interesa esta cuestión es en la medida en que el mundo actual y sus configuraciones culturales generan habitualidades reflexivas según la cual los sujetos se ven confrontados consigo mismos de un modo constante y sin excepción. La potencia reflexiva viene elevada a actualidad de un modo involuntario, no dispuesto libremente. Los sujetos se ven expuestos a una continua pre-

\footnotetext{
${ }^{38}$ HUSSERL, Edmund. Las crisis de las ciencias europeas, op. cit., p. 195
} 
sencia de sí, reflejados constantemente en los objetos que habrían de ocupar su atención. Con ello no está en juego una crítica a un modelo de cultura, sino la posibilidad misma de la filosofía. Es por ello que un problema que parecería caer del lado de la psicología, o de la sociología, debe interesar también a la fenomenología. Si bien una reflexión en sentido fenomenológico es por principio siempre posible, no es impensable un mundo en el que esta posibilidad sea arruinada desde la raíz. Pienso en un tipo de reflexión psicológica que no encontrase otra salida que ulteriores reflexiones sobre reflexiones en un círculo vicioso $^{39}$. Este problema se vislumbra al contemplar personas cada vez más preocupadas por sí mismas, por su rendimiento laboral, su imagen pública, por su consideración social, etc. Podríamos hablar entonces de una reflexividad patológica que suplanta y obstaculiza la reflexión fenomenológica. Tal patología implicaría que toda reflexión ulterior de un sujeto sobre su propia condición reflexiva condujese a un regreso al infinito en un sentido no solamente lógico, sino también emocional. Una atención creciente al propio bienestar o malestar, al equilibrio interior, al crecimiento personal. En suma, una atención hacia una supuesta interioridad que distorsionaría la atención normal del sujeto hacia la trascendencia del mundo al tiempo que impediría la revelación de la inmanencia trascendental por parte de un tipo de reflexión no psicológica.

Como hemos visto, la vida de conciencia implica de modo pasivo una remisión genitiva a un polo subjetivo, a un yo que se mantiene idéntico a lo largo y ancho de la corriente de vivencias. La apropiación de la vida de conciencia no exige por tanto un acto voluntario, un acto de fundación. Ésta remite pasivamente a un sujeto que concentra su vida, que es el sujeto de la misma, vivida en un modo genitivo: la vida vivida en cada caso como la mía. Esta polaridad no necesita de una intervención activa por parte del sujeto: es fruto de síntesis pasivas. Pero no se puede dejar de notar que este polo subjetivo es también un centro de afecciones, y que por lo tanto su identidad se envuelve, a medida que el sujeto vive y hace su historia, de habitualidades en las que éste gana una forma concreta. En principio, este envolvimiento no consiente rotura alguna. Sin embargo, en casos límite, al sujeto puede perder el sentimiento de esta

\footnotetext{
${ }^{39}$ No es un fenómeno extraño hoy en día el de reflexiones sobre reflexiones que acaban por perder su finalidad y quedan atrapadas sin solución de continuidad. Pienso en reflexiones del tipo: «¿por qué pienso esto?», «¿por qué estoy pensando en mi propio pensamiento?», «debería de dejar de pensar en mi propio pensamiento».
} 
identidad o de coincidencia entre el polo subjetivo de su vida y las habitualidades que lo definen como un yo fáctico. El sujeto puede entonces concentrar su capacidad reflexiva en tratar de recuperar esta unidad perdida. Es decir, el sujeto puede tratar de captar el polo subjetivo que late en la corriente de vivencias no por una voluntad de conocimiento, sino por una necesidad de reconocimiento. Hablaríamos entonces de una conciencia desventurada. Una errónea vía a la hora de llevar a cabo el clásico imperativo «conócete a ti mismo».

Husserl ya comprendió la reflexión como una actividad antinatural, pero consiguió sublimar este carácter hacia un fin creativo, en una entrega que no envolviese al fenomenólogo en un círculo encantado ${ }^{40}$. Con todo, no dejó de reconocer los peligros de cierta circularidad, así como la posibilidad de que la reflexión quedase atrapada en una regresión al infinito. Esta misma idea, como ya hemos anotado, aparecerá con un doble carácter a lo largo de su obra: por un lado, el peligro de quedar atrapada la reflexión en una espiral sin fin; por el otro, la reflexión como una exploración y una explicitación de la vida in infinitum. La antinaturalidad de la reflexión se volvería así solidaria de la vida de conciencia en un sentido productivo. Pero el peligro de que esta antinaturalidad sea naturalizada no deja de amenazar. Es así que en cierto modo la hiperreflexividad se define de un modo no extremadamente diferente de la reflexión fenomenológica: se trata de volver conscientes, para explicitarlos, los rendimientos intencionales del campo trascendental, para comprender el origen del mundo como origen de todo horizonte de sentido. La hiperreflexividad no sería sino la excesiva conciencia de las espontaneidades subjetivas y sus correlaciones asociativas, aquellas que vinculan la percepción, la memoria, el juicio, los sentimientos, la fantasía, etc. ${ }^{41}$

El caso de la hiperreflexividad es aquel en el que el yo puro (aquel que puede entrar y/o salir de escena) pasa a ser consciente de un modo constante,

\footnotetext{
${ }^{40}$ Cfr, HUSSERL, Edmund, Erste Philosophie (1923/24), Zweiter Teil, Den Haag, Martinus Nijhoff, 1973, 1959, lección 45.

${ }^{41}$ «Se define la autoconciencia hiperreflexiva como un exceso de conciencia que toma como objeto a uno mismo, sea el yo o cualquier evento privado (experiencia, sentimientos, pensamientos, etc.) o incluso el propio cuerpo. La cuestión es que aspectos de uno normalmente implícitos o en su caso objeto ya de cierta reflexión, se convierten en explícitos, al punto de ocupar buena parte de la atención y así copar el campo de la conciencia. Se trata de una excesiva presencia de uno para sí mismo que lleva a alterar, si es que no perturbar, el contacto con el mundo dado por los asuntos prácticos de la vida. El criterio de «exceso» lo da la disfuncionalidad que llega a producir, dejando por tanto de ser funcional, adaptativa y práctica, para ser disfuncional y hasta patógena. Cuando la reflexividad deja de resolver el problema de que se ocupa convirtiéndose ella misma en un problema, siendo ya más parte del problema que de la solución, estamos en presencia de una autoconciencia hiperreflexiva patógena». PÉREZ ÁLVAREZ, Marino, Las raíces de la psicopatología moderna, Madrid, Pirámide, 2012, p. 50
} 
denunciando la polaridad subjetiva de cada vivencia hasta tal punto de destruir su carácter operativo. El análisis fenomenológico refiere a un yo que vive en sus actos, del que irradian los actos intencionales que nos sitúan ante los objetos del mundo. Este yo opera en muchos casos de modo latente, pero puede ser captado por una reflexión particular. En el caso de la hiperreflexividad, este yo latente estaría constantemente destacado. De otro modo, la atención estaría dirigida no al objeto intencional que un acto revela, sino al carácter sujetivo del mismo, es decir, al carácter genitivo de cada vivencia (soy yo el que ahora percibo, soy yo el que ahora recuerdo, etc.). El yo en estos casos se siente excesivamente comprometido en cada uno de sus actos, responsable de los mismos, lo que hace que su actividad se agote en esta inminencia de sí que satura e impide el cumplimiento natural de la actividad intencional. La cuestión, de nuevo, pasa por no comprender este problema como accidental, propio de un sujeto aislado, sino como estructural de una cultura y un mundo que condiciona, en virtud de su modo de afectar al sujeto, las habitualidades en las que éste forma su historia y forja su identidad. La reflexión, con ser una actividad antinatural, se genera en las inflexiones a las que la vida concreta nos aboca. Se definen nuestras posiciones dóxicas con arreglo a afecciones socialmente estructuradas y en donde no solo cuentan los otros sujetos y sus motivaciones, sino también las costumbres, las obras culturales, las instituciones, etc ${ }^{42}$. El problema no está puesto, repetimos, en circunstancias personales, sino en motivaciones intersubjetivas que exceden el campo de la psicología y plantean problemas filosóficos.

El hecho de que los temas aquí tratados - los diferentes niveles de subjetividad, la génesis de las habitualidades y los tipos de reflexión- hayan quedado abiertos en la obra de Husserl animan a que la fenomenología actual, ante problemas que, a mi juicio no provienen tanto de una crisis de las ciencias como de una crisis de la cultura, elabore programas rigurosos y que se atengan a la cosa misma, en la medida en que está en juego la posibilidad misma de filosofar. Se trata de abordar un peligro constante en la historia de la humanidad, que es la deriva del sano asombro ante el mundo y de la extrañeza filosófica en enajenación personal y delirio.

${ }^{42}$ cfr, HUSSERL, E. Ideas II. Op. cit. , §56 
Los problemas aquí esbozados no son nuevos. De hecho la fenomenología ha impregnado desde bien pronto aquellas investigaciones y disciplinas que podemos englobar bajo el rótulo general de psicopatología. Aquí, sin pretender establecer el mejor de los accesos a esta relación interdisciplinar, hemos querido presentar, sencillamente, una introducción que seguramente será coincidente en muchos puntos con las ya existentes y en desarrollo. Con ello no he querido sino justificar por cuenta propia la posibilidad y la pertinencia de una investigación fenomenológica referida a la constitución comunitaria de habitualidades intersubjetivas como la expuesta ${ }^{43}$. Partiendo de habitualidades que, generadas en el mundo de la vida, calan hasta la subjetividad en sentido trascendental, he querido indicar la posibilidad de que la fenomenología tenga en consideración la situación histórica en la que el fenomenólogo vive. Husserl mismo fue sensible a esta consideración, como se ve en su libro La crisis de las ciencias europeas y la fenomenología trascendental, donde se relacionan estrechamente la circunstancia científica de una época y sus consecuencias para la posibilidad de la filosofía ${ }^{44}$. En este caso hemos querido apuntar la idea de que una sociedad como la nuestra se caracteriza por una hiperreflexividad institucionalizada en diferentes prácticas cotidianas que de igual modo obligan a pensar la posibilidad de la filosofía. A mi juicio esta circunstancia es también objeto de la fenomenología, si bien cae fuera del método específico de las reducciones fenomenológicas. Se abre con ello una vía, la de una fenomenología entendida como crítica de la cultura. Una fenomenología no solo entendida como ciencia rigurosa según una voluntad de conocimiento, sino también según una voluntad de salud.

\footnotetext{
${ }^{43}$ Por motivos principalmente referidos a las dimensiones de este trabajo, me he limitado a la fenomenología de Husserl como primera justificación de una relación entre la fenomenología y la psicopatología. En trabajos posteriores trataré de dar cuenta de estas relaciones con una mayor atención a los trabajos que parten de la psicopatología como los de Jaspers, Binswanger, Minkowski, etc.

${ }^{44}$ Husserl de hecho relaciona directamente la crisis de las ciencias con una crisis de la cultura. En su caso, percibimos su interés por considerar la deriva histórica de las ciencias en una sentencia muy conocida: «Tomamos nuestro punto de partida en el ingreso de un cambio en la valoración general respecto de las ciencias, a fines del último siglo. No concierne a su carácter científico sino a lo que la ciencia en general había significado y puede significar para la existencia humana. La exclusividad con que en la segunda mitad del siglo XIX, la total visión del mundo de los seres humanos modernos se deja determinar y cegar por las ciencias positivas y por la "prosperity" de que son deudores, significó un alejamiento indifierente de las preguntas que son decisivas para una auténtica humanidad. Meras ciencias de hechos hacen meros seres humanos de hechos» (HUSSERL, E. La crisis de las ciencias europeas y la fenomenología trascendental, op. cit., pp. 49-50)
} 


\section{BibLIOGRAFÍA}

HUSSERL, Edmund. Investigaciones lógicas, Revista de Occidente, Madrid, 1967

- Grundprobleme der Phänomenologie 1910/11, Martinus Nijhoff, Den Haag, 1977

- Ideas relativas a una fenomenología pura y a una filosofía fenomenológica. Libro primero: Introducción general a la fenomenología pura, Fondo de Cultura Económica, México, 2013

- Ideas relativas a una fenomenología pura y una filosofía fenomenológica. Libro Segundo: Investigaciones fenomenológicas sobre la constitución, Fondo de Cultura Económica, México, 2005

- Die "Bernauer Manuskripte" über das Zeitbewußtsein (1917/18). Kluwer Academic Publishers, Dordrecht, Netherlands, 2001

- Erste Philosophie (1923/24), Zweiter Teil, Martinus Nijhoff, Den Haag, 1973, 1959

- Phänomenologische Psychologie. Vorlesungen Sommersemester 1925. Martinus Nijhoff, Den Haag, 1966

- Meditaciones cartesianas, Fondo de Cultura Económica, México, 2005

- Zur Phänomenologie der Intersubjetivität (1929-1935). Martinus Nijhoff, Den Haag, 1973

- Las crisis de las ciencias europeas y la fenomenología trascendental. Prometeo libros, Buenos Aires, 2008

MARBACH, Eduard, Das Problem des Ich in der Phänomenologie Husserl, Martinus Nijhoff, Den Haag, 1974

PÉREZ ÁlVAREZ, Marino, Las raíces de la psicopatología moderna, Pirámide, Madrid, 2012

TAGUCHI, Shigeru. Das Problem des ,Ur-Ich' bei Edmund Husserl, Springer, Dordrecht, 2006 